Received 00th January 20xx, Accepted 00th January $20 x x$ DOI: $10.1039 / x 0 x \times 00000 x$

\title{
Beyond the Active Site: Mechanistic Investigations of the Role of the Secondary Coordination Sphere and Beyond on Multi-Electron Electrocatalytic Reactions and Their Relations Between Kinetics and Thermodynamics
}

\begin{abstract}
Vincent C.-C. Wanga
The development of an electrocatalyst with a rapid turnover frequency, low overpotential and long-term stability is highly desired for fuel-forming reactions, such as water splitting and $\mathrm{CO}_{2}$ reduction. The findings of the scaling relationships between the catalytic rate and thermodynamic parameters over a wide range of electrocatalysts in homogeneous and heterogeneous systems provide useful guidelines and predictions for designing better catalysts for those redox reactions. However, such relationships also suggest that a catalyst with a high catalytic rate is typically associated with a high overpotential for a given reaction. Inspired by enzymes, the introduction of additional interactions through the secondary coordination sphere beyond the active site, such as hydrogen-bonding or electrostatic interactions, have been shown to offer a promising avenue to disrupt these unfavorable relationships. Herein, we further investigate the influence of these cooperative interactions on the faster chemical steps, in addition to the rate-limiting step widely examined before, for molecular electrocatalysts with the structural and electronic modifications designed to facilitate the dioxygen reduction reaction, $\mathrm{CO}_{2}$ reduction reaction and hydrogen evolving reaction. Based on the electrocatalytic kinetic analysis, the rate constants for faster chemical steps and their correlation with the corresponding thermodynamic parameters are evaluated. The results suggest that the effects of the secondary coordination sphere and beyond on these fuel-forming reactions are not necessarily beneficial for promoting all chemical steps and no apparent relation between rate constants and thermodynamic parameters are found in some cases studied here, which may implicate the design of electrocatalysts in the future. Finally, these analyses demonstrate that the characteristic features for voltammograms and foot-of-the-waveanalysis plots are associated with the specific kinetic phenomenon among these multi-electron electrocatalytic reactions, which provides a useful framework to probe the insights of chemical and electronic modifications on the catalytic steps quantitatively (i.e. kinetic rate constants) and to optimize some of critical steps beyond the rate-limiting step.
\end{abstract}

\section{Introduction}

The development of electrocatalysts for fuel-forming reactions, such as water splitting and $\mathrm{CO}_{2}$ reduction, plays a vital role for future sustainable and renewable energy. A desired electrocatalyst is expected to have a high turnover frequency (TOF), small overpotential and high stability (turnover number). In recent years, a great amount of research efforts have been made to understand operating principles and detailed mechanisms toward these aims in both homogeneous and heterogeneous systems. ${ }^{1-3}$ One of important findings is the scaling relationship between the catalytic rates and thermodynamic values (e.g. overpotential or binding energy between substrate and catalyst surface) found in both systems, which allows to predict the trend of electrocatalytic activity

\footnotetext{
a. Program of Physical Chemistry, Department of Chemistry - Ångström Laboratory,
} Uppsala University, Uppsala 75120 Sweden.E-mail:vincentwang64@yahoo.com. over catalysts and provides guidelines for rational designing of better electrocatalysts. ${ }^{1-3}$ Nevertheless, such relationship implies certain constraints on the optimization of catalysts, where changes made for one step of the catalytic cycle is more favorable for the overall reaction, but results in disfavoring of other steps (the Sabatier principle). This trade-off leads to a high catalytic rate that is typically associated with a high overpotential in homogeneous molecular systems.

Take the reaction through the simple EC mechanism (E: electron transfer step and C: chemical step) of a molecular electrocatalyst as an example shown in Fig 1a. In general, to modulate the catalytic activity of a catalyst, the electronic and chemical structure of the active site could be tuned directly through a modification of electron-withdrawing or electrondonating groups. For example, the substitution with an electron-withdrawing group is expected to raise the reduction potential of a catalyst through the inductive effect to facilitate the E step. Therefore, as shown in Fig 1b, a lower overpotential 
a

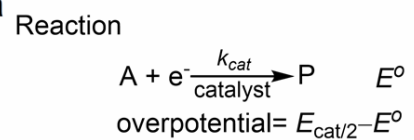

EC mechanism

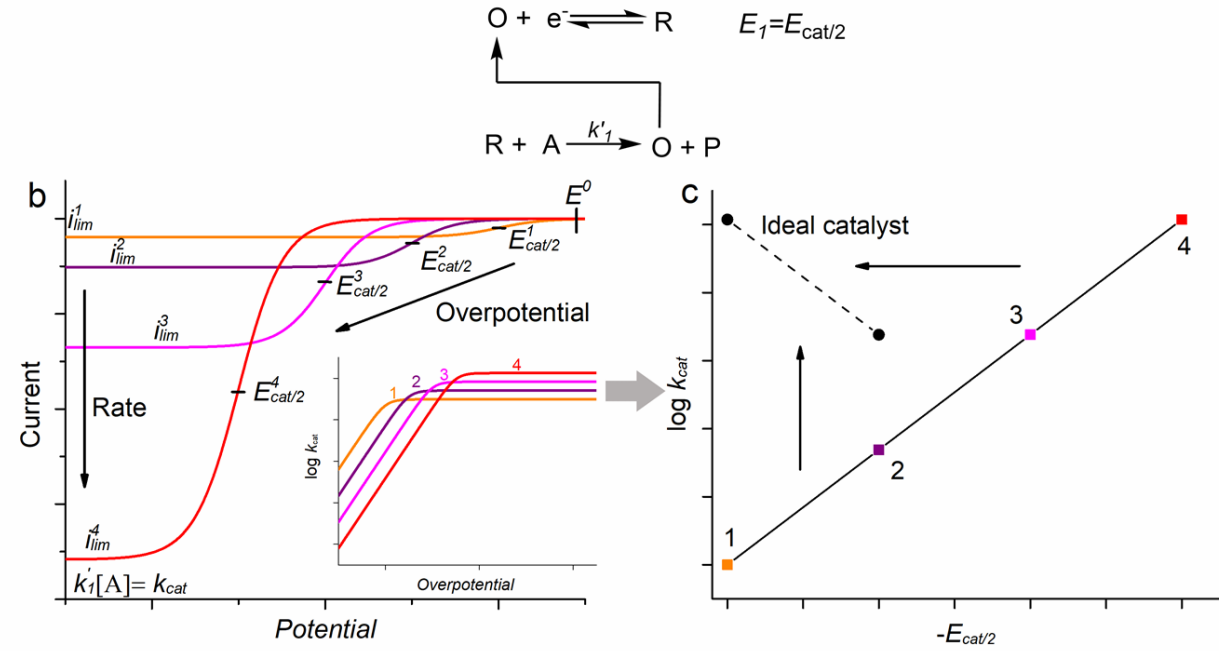

Fig 1. The illustration of a scaling relationship for the EC mechanism in the homogeneous molecular system. In the reaction scheme, A is a reactant and $\mathrm{P}$ is a product. $\mathrm{O}$ and $\mathrm{R}$ indicate the oxidized and reduced state of a catalyst respectively. $E^{O}$ refers to the standard reduction potential for the given reaction and $E_{1}$ is the reduction potential of a catalyst. For the EC mechanism, $E_{1}$ is equal to $E_{c a t / 2}(v i d e \text { infra })^{8}$ and $k_{1}^{\prime}$ is the second order rate constant. Fig $1 \mathrm{~b}$ shows typical electrocatalytic voltammograms for homogeneous molecular catalysts in a pure kinetic EC condition and their corresponding catalytic Tafel plots (inset). Fig $1 \mathrm{c}$ reveals a scaling relationship plot extracted from Fig $1 \mathrm{~b}$.

is required with respect to the standard reduction potential, $E^{o}$ for a given reaction. However, in the meantime, this substitution introduces a counter-balancing effect on the $C$ step (e.g. a positively charged proton transfer) due to the lower electron density of the active site, which yields a lower catalytic rate. Overall, such modification yields an electrocatalyst with a low overpotential but slow TOF, as illustrated in the orange line of Fig 1b. In contrast, the substitution of an electron-donating group leads to the opposite outcome (i.e. a high overpotential but faster catalytic rate as shown in the red line of Fig $1 \mathrm{~b}$ ). Therefore, the development of new approaches to address this conundrum plays an important role in further facilitating fuelforming reactions.

Inspired by [FeFe] hydrogenases that perform the hydrogen evolving reaction with a minimal overpotential and high TOF, ${ }^{4}$ it has been recently demonstrated that the addition of specific functional groups to biomimetic HER catalysts at the secondary coordination sphere (SCS) to serve as proton relays near the active site can greatly facilitate fuel-forming reactions without the need to build a large overpotential into the design of the catalyst. ${ }^{3,5-7}$ In other words, the secondary coordination sphere, treated as an additional dimension, can be further introduced to tailor catalytic reactivity through cooperative interactions, such as electrostatic or hydrogen-bonding interactions beyond the active site. Nevertheless, so far, most reports focused on the rate-limiting step and the relationship between its kinetic rates and thermodynamic parameters (such as the reduction potential or $p K_{a}$ ), without considering that most reactions of interest are multi-electron and multi-step reactions. It is important to further understand how the SCS affects other critical faster chemical steps in a catalytic cycle and their relationships between kinetic and thermodynamic properties, which could implicate overall performance, such as undesired side effects on the rate. The findings of key features and correlation between elementary reaction steps influenced by the SCS and beyond through a high degree of synthetic tunability of molecular catalysts can further provide the essence of design principles for homogeneous and heterogeneous systems in order to circumvent if not overcome the unfavourable correlation relationships.

Here, we examine molecular electrocatalysts for oxygen reduction reaction (ORR), carbon dioxide reduction reaction (CRR) and hydrogen evolving reaction (HER) reported in literature exploiting the effect of the SCS to mediate the reactions. The kinetic models for multi-electron electrocatalytic reactions, developed by Savéant and Costentin, ${ }^{8}$ will be applied as an essential tool for kinetic analysis to determine kinetic rate constants and explore connections between kinetic and thermodynamic properties among reaction steps. Particularly, in some scenarios, the rate constant for the faster chemical step rather than the rate-limiting step is determined by the foot of the wave analysis (FOWA) of electrocatalytic voltammograms, which is sometimes misinterpreted in the community. ${ }^{8-10}$ 

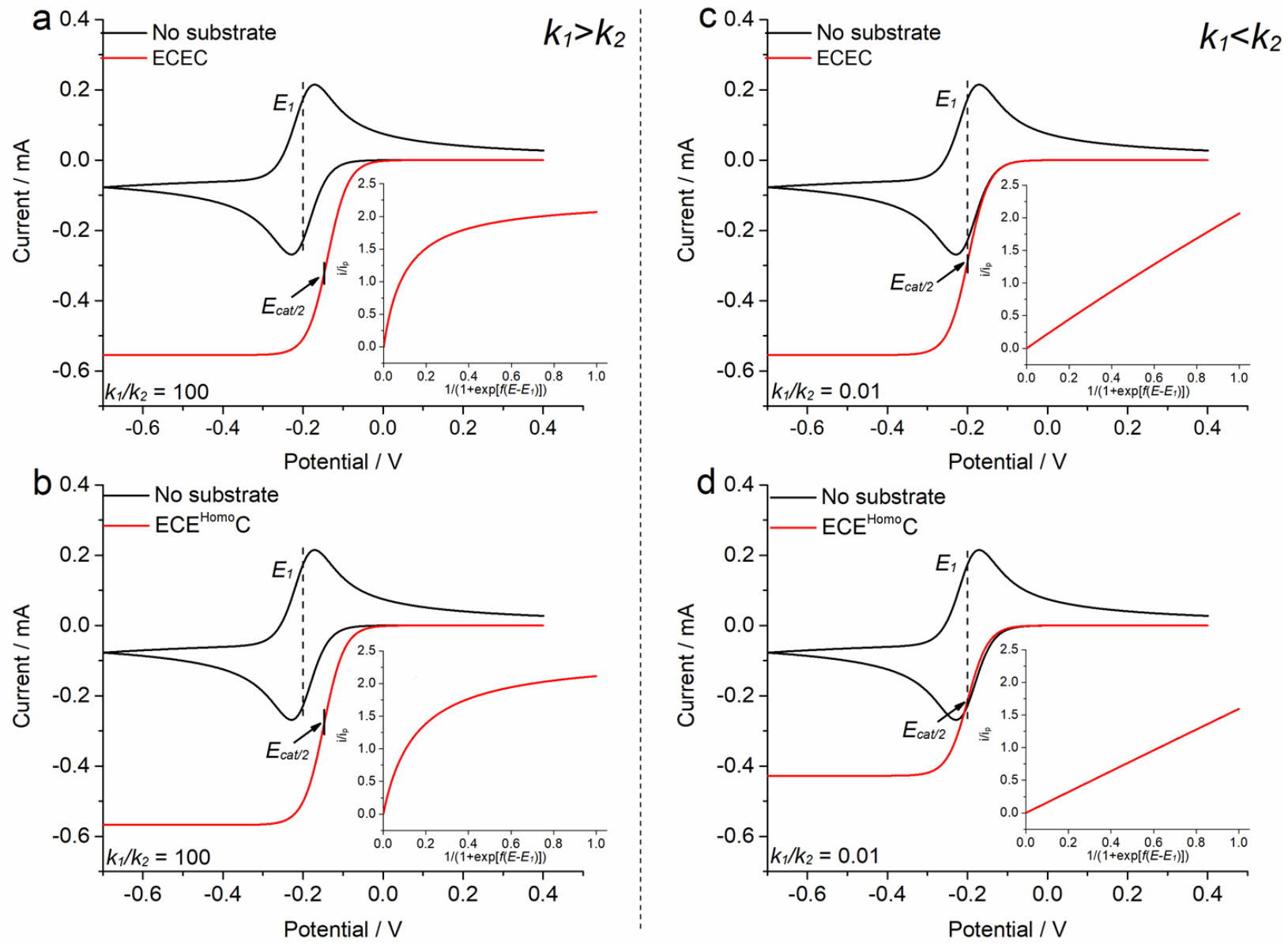

Fig 2. Electrocatalytic voltammograms and corresponding FOWA plots (inset figures) for ECEC and ECE ${ }^{\text {Homo }}{ }^{\circ}$ mechanism. Two different conditions for the second electron transfer are considered, ECEC and ECE ${ }^{\mathrm{Hom}}{ }^{\circ} \mathrm{C}$. For Fig $2 \mathrm{a}$ and $2 \mathrm{~b}$, (scenario $\mathrm{A}, k_{1}>k_{2}$ ), the rate constants, $k_{1}=100 \mathrm{~s}^{-1}$ and $k_{2}=1 \mathrm{~s}^{-1}$ were used to obtain voltammograms. For Fig $2 \mathrm{c}$ and $2 \mathrm{~d}$, (scenario $\mathrm{B}, k_{1}<k_{2}$ ), the rate constants, $\mathrm{k}_{1}=1 \mathrm{~s}^{-1}$ and $\mathrm{k}_{2}=100 \mathrm{~s}^{-1}$ were used. The rest parameters for simulations are $E_{1}=-0.2 \mathrm{~V}, E_{2}=0 \mathrm{~V}$ and the diffusion coefficient for catalysts, $10^{-5} \mathrm{~cm}^{2} / \mathrm{s}$. For clarity, in the black line, only the redox peak for $E_{1}$ was presented here and electrode kinetics follow the Nernstian behavior.

\section{Results}

The general guidelines for determining the kinetic and thermodynamic values from electrocatalytic voltammograms

To evaluate kinetic and thermodynamic properties of a given electrocatalyst, the electrocatalytic voltammetry is a widely used tool. ${ }^{11}$ When a canonical electrocatalytic S-shape voltammogram is achieved as shown in Fig 1 or 2, the catalytic potential, $E_{c a t / 2}$ is defined at a half-wave of the potential ${ }^{12}$ and the catalytic rate (i.e. TOF) or observed rate constant, $k_{o b s}$ for the overall reaction is determined from the limiting current, $i_{\text {lim }}$ according to eq (1) or (2). ${ }^{13,14}$

$$
i_{\text {lim }}=n F S C^{*} \sqrt{D_{c a t} k_{o b s}}(1), \frac{i_{l i m}}{i_{p}}=2.24 n \sqrt{\frac{R T}{F v}} \sqrt{k_{o b s}}
$$

where $i_{p}$ refers to the peak current for a redox couple of a catalyst associated with catalysis in the absence of substrate. $R$ : ideal gas constant, $T$ : absolute temperature, $F$ : Faraday constant, $S$ : electroactive area, $D_{\text {cat }}$ : the diffusion coefficient for the catalyst, $C^{*}$ : a total concentration of electrocatalysts, $n$ : the number of electrons involved with the reaction.

Compared to eq 1 , the determination of $k_{o b s}$ by eq 2 is more straightforward due to no prior knowledge for the electroactive area and the diffusion coefficient of the catalyst. If the S-shape voltammogram is not achievable, due to side phenomena such as substrate depletion, the foot of the wave analysis can be applied to determine the catalytic rate constant. ${ }^{15}$ However, for multi-electron redox reactions, it was found that, in some scenarios, the rate constant determined by FOWA is the faster chemical step rather than the rate-limiting step as expected from the EC mechanism. ${ }^{8-10}$

Take the most likely-encountered ECEC reaction mechanism with $E_{1}<E_{2}$ in the reduction direction (reaction schemes as shown in Table 1) as examples shown in Fig 2 where scenario $\mathbf{A}$ refers to $k_{1}>k_{2}$ and scenario $\mathrm{B}$ refers to $k_{1}<k_{2}$. Since the second electron transfer can either occur through the interfacial electron transfer between an electrode and catalysts or through 
rapid homogeneous electron transfer among catalysts, the ECEC and $\mathrm{ECE}^{\mathrm{Homo}}{ }^{\circ} \mathrm{C}$ mechanism are discussed here. For a Sshape electrocatalytic voltammogram, typically, the rate constant for the rate-limiting step is directly estimated by $i_{\text {lim }}$ (e.g. $k_{o b s} \approx k_{2}$ for scenario $\mathrm{A}$, and $k_{o b s} \approx k_{1}$ for scenario $\mathrm{B}$ ) since the overall rate is limited by the slowest step. Alternatively, for scenario $\mathrm{A}$, a more precise determination of $k_{1}$ and $k_{2}$ can be achieved by the combination of eq (T2) and eq (T3) for ECEC or eq (T6) and eq (T7) for $\mathrm{ECE}^{\mathrm{Homo}}{ }^{\circ} \mathrm{C}$ as equations summarized in Table $1 .{ }^{10}$ For most electrocatalytic reactions, it is expected that more than one elementary step for chemical reaction, not as shown in the reaction scheme of Table 1 , are involved (such as fuel-forming reactions discussed later). Nevertheless, a single apparent rate constant can be used to represent a collective combination of all elementary chemical steps involved. Therefore, the rate constants, $k_{1}$ and $k_{2}$ determined by kinetic schemes shown in Table 1 can be treated as the apparent rate constant.

For the feature of a FOWA plot, the general I-V relationship depicted for ECEC (eq T1 in Table 1) can be approximated to eq 3 when scenario A occurs with the first formal potential, $E_{1}$ that is more negative than $E_{2}(\sim 150 \mathrm{mV}) \cdot{ }^{9}$ For $\mathrm{ECE}^{\mathrm{Hom}}{ }^{\circ} \mathrm{C}$, the general equation (eq T5) can be rearranged as eq 4. (The detailed derivation can be found in $\mathrm{SI}$ and ref 8,9 ).

$$
\begin{gathered}
\boldsymbol{i}=\frac{2 F S C^{*} \sqrt{D_{c a t} k_{1}}}{1+\varepsilon_{1}+\frac{\sqrt{k_{1}}}{\sqrt{k_{2}}}} \text { (3) for ECEC } \\
\boldsymbol{i}=\frac{\boldsymbol{F} S C^{*} \sqrt{2 D_{c a t} k_{1}}}{1+\varepsilon_{1}+\left(\frac{\sqrt{\frac{2 k_{1}}{k_{2}}-1}}{2-\frac{k_{2}}{k_{1}}}\right)} \text { (4) for ECE }{ }^{\text {Homo }} \text { C }
\end{gathered}
$$

$\varepsilon_{1}=\exp \left[f\left(E-E_{1}\right)\right]$ and $E$ : electrode potential

Therefore, when plotting $i$ against $\left(1+\varepsilon_{1}\right)$ (i.e. FOWA plot), the curve shape of a FOWA plot is obtained instead of a straight line expected from the EC mechanism due to the additional term, $\left(\sqrt{k_{1}} / \sqrt{k_{2}}\right)$ or $\left[\left(\sqrt{2 k_{1} / k_{2}}-1\right) /\left(2-k_{2} / k_{1}\right)\right]$ in the denominator from eq 3 and eq 4 respectively. ${ }^{9}$ As a result, the rate constant determined by a FOWA plot for scenario $\mathbf{A}$ is $k_{1}$ (i.e. the faster chemical step) rather than the rate-limiting kinetic constant, $k_{2}$ that should be determined by $i_{\text {lim. }}{ }^{9}$ In comparison, for scenario $\mathbf{B}$, the approximate equations, eq 5 and 6 are obtained respectively for ECEC and ECE ${ }^{\text {Homo }}{ }^{\circ}$. Therefore, the FOWA plot is expected to reveal a straight line and the rate-limiting kinetic constant is obtained from the slope.

$$
\begin{gathered}
i=\frac{2 F S C^{*} \sqrt{D_{c a t} k_{1}}}{1+\varepsilon_{1}}(5) \text { for ECEC } \\
i=\frac{F S C^{*} \sqrt{2 D_{c a t} k_{1}}}{1+\varepsilon_{1}} \text { (6) for ECE }{ }^{\text {Homo }} \mathrm{C}
\end{gathered}
$$

The characteristic features of electrocatalytic voltammograms for these mechanisms and the corresponding FOWA plots are demonstrated in Fig 2 and summarized in Table 1 . The key different features between scenario $\mathbf{A}$ and $\mathbf{B}$ are that an anodic shift of the $E_{\text {cat } / 2}$ value of electrocatalytic voltammograms with respect to $E_{1}$ and the curve shape of the corresponding FOWA plots are observed for scenario $\mathbf{A}$ as shown in Fig 2a-b. In other words, as the concentration of substrate $A$ increases, the anodic shift of a voltammogram is expected for $k_{1}>k_{2}$. It is worth noting that even in the presence of substrate depletion, such key features still can be observed since negligible side phenomena are expected in the region of low overpotential. ${ }^{9}$ These results suggest that the feature of FOWA plots can be used to probe mechanistic insights. In addition, the $k_{1}$ value determined by FOWA is sensitive to the difference between $E_{1}$ and $E_{2}$ (for ECEC) and the ratio of $k_{1} / k_{2} \cdot{ }^{9}$ Alternatively, the $k_{1}$ value can be estimated through $E_{c a t / 2}$ that varies with the ratio of $\left(\sqrt{k_{1}} / \sqrt{k_{2}}\right)$ (Table 1 ) in the scenario A. Therefore, Table 1 is used as guidance to characterize the reaction mechanism and obtain the corresponding apparent rate constant in the following case studies.

\section{Case studies}

\section{Case A: Oxygen reduction reaction (ORR)}

Recently, a series of $\mathrm{Fe}$ tetraphenylporphyrin (FeTPP) derivatives has been prepared by the Mayer group to investigate their catalytic mechanisms for ORR and the effect of the SCS through judicious designs of porphyrin ligands. ${ }^{16-20}$ All reported rate constants for these complexes were determined by FOWA, which they referred to as the rate-limiting step for the first proton transfer as shown in Scheme 1 (eq (5) ORR) following sequential EC steps. However, re-examinations of voltammograms and FOWA plots suggest that the rate constant determined by FOWA is not the rate-limiting step and instead should be assigned to the first fast chemical step as suggested in the Table 1. The most prominent evidence to support this new interpretation is that all FOWA plots reported from ref 1618 have a curve shape. Furthermore, the appreciable extent of the anodic shift of voltammograms (or the anodic shift of the pseudo $E_{\text {cat } / 2}$ ) was observed as the concentration of the proton source was increased (i.e. the pseudo first order rate constant increased). These features clearly indicate that the rate constant determined by FOWA is not the rate-limiting step. In stark contrast, the same FeTPP complex was also shown to be capable of catalyzing the reduction of $\mathrm{CO}_{2}$ to $\mathrm{CO}$, in which the FOWA plot revealed a straight line (e.g. Ref 16 for ORR vs ref 21 for (RR) because $k_{1}^{C R R}$ is much smaller than $k_{2}^{C R R}$, which indicates the rate constant determined by FOWA for CRR is the rate-limiting step. (vide infra)

Therefore, the rate constant determined by FOWA for ORR meditated by the Fe(TPP) complex is re-assigned as an apparent rate constant, $k_{1}^{O R R}$ that represents a collection of faster chemical steps between the first and second $E$ step as shown in Scheme 1 (i.e. the combination of eq (4)-(5) ORR). The $k_{1}^{O R R}$ value is likely to be greatly affected by the first proton transfer step (eq (5) ORR) as suggested. ${ }^{16,18,19}$ For the rate constant of the ratelimiting step, $k_{2}^{O R R}$, this value is proposed to be associated with the reaction of the $\mathrm{O}-\mathrm{O}$ bond breaking step (eq (7) ORR), which thereby should be determined by $i_{\text {lim }}$. Reanalysis of voltammograms from ref 16 to determine $k_{2}^{O R R}$ through $i_{\text {lim }}$ afforded the third order rate constant, $k_{o r}, 9.6 \times 10^{5} \mathrm{M}^{-2} \mathrm{~s}^{-1}$ 
Table 1. Summary of key features for ECEC mechanism.

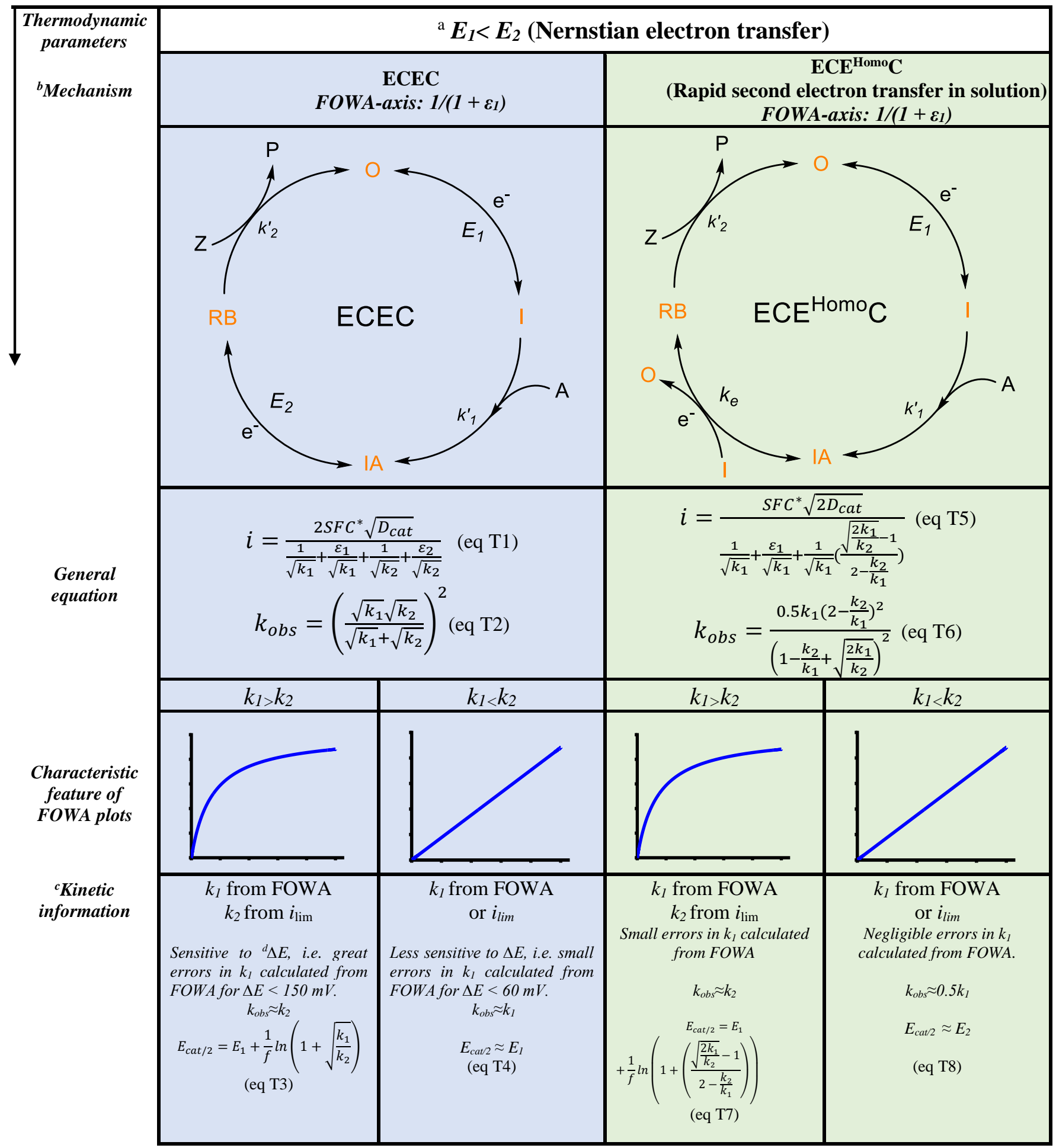

a For ECEC with $E_{1}>E_{2}$, results between $k_{1}>k_{2}$ and $k_{1}<k_{2}$ are exchanged since the ECEC mechanism can be regarded as two individual EC reactions. ${ }^{9}$

${ }^{\mathrm{b}} \boldsymbol{k}_{\mathbf{1}}^{\prime}$ and $\boldsymbol{k}_{\mathbf{2}}^{\prime}$ are the second-order rate constants for the first and second chemical steps respectively. The pseudo first order rate constants $\left(\boldsymbol{k}_{\mathbf{1}}=\boldsymbol{k}_{\mathbf{1}}^{\prime} \boldsymbol{C}_{\boldsymbol{A}}^{\boldsymbol{o}} ; \boldsymbol{k}_{\mathbf{2}}=\boldsymbol{k}_{\mathbf{2}}^{\prime} \boldsymbol{C}_{\boldsymbol{Z}}^{\boldsymbol{o}}\right)$ are discussed here since the concentration of substrate is much higher than the one of catalysts. ${ }^{8} E_{1}$ and $E_{2}$ refer to the formal reduction potential for the first and second electron transfer respectively. $k_{e}$ indicates the rapid homogeneous electron transfer between catalysts. The orange color refers to the state of a catalyst in the catalytic cycle. $\mathrm{O}$ : oxidized state, I: one-electron reduced state, IA and RB: one-electron and two-electron reduced substrate-bound intermediate state. $\mathrm{A}$ and $\mathrm{Z}$ : co-substrates, $\mathrm{P}$ : product. $\quad{ }^{\mathrm{c}}$ ref $9 .{ }^{\mathrm{d}} \Delta E=\left|E_{1}-E_{2}\right|$. 


$$
\begin{aligned}
\mathrm{O}_{2}+4 \mathrm{e}^{-}+4 \mathrm{HA} & \stackrel{\mathrm{TPP}-\mathrm{Fe}}{\longrightarrow} 2 \mathrm{H}_{2} \mathrm{O}+4 \mathrm{~A}^{-} & & \text {(1) ORR } \\
\mathrm{HA} & \rightleftharpoons \mathrm{H}^{+}+\mathrm{A}^{-} & \mathrm{K}_{a} & \left.{ }{ }^{2}\right)_{O R R}
\end{aligned}
$$

Mechanism

$$
\begin{aligned}
& \mathrm{TPP}-\mathrm{Fe}(\mathrm{III})+\mathrm{e}^{-} \rightleftharpoons \text { TPP-Fe(II) } \\
& \text { TPP-Fe(II) }+\mathrm{O}_{2} \stackrel{k_{1\left(\mathrm{O}_{2}\right)}}{\longrightarrow} \text { TPP-Fe(III)-O- }
\end{aligned}
$$

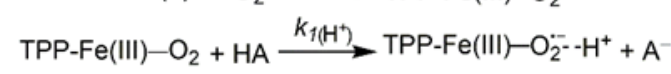

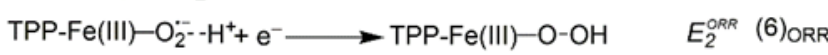

$$
\begin{aligned}
& \text { TPP-Fe(III)-O-OH+ HA } \underset{\text { Rate-limiting step }}{\stackrel{k_{2\left(\mathrm{H}^{+}\right)}}{\longrightarrow}} \text { TPP-Fe(V)=O + } \mathrm{A}^{-}+\mathrm{H}_{2} \mathrm{O} \\
& (7)_{\mathrm{ORR}} \Rightarrow k_{2}^{\mathrm{ORR}} \\
& E_{1}^{\text {ORR }}(3)_{\text {ORR }} \\
& \mathrm{KO}_{2} \quad(4)_{\mathrm{ORR}} \\
& \text { (5) ORR } k_{1}^{O R R} \\
& \text { (8) }
\end{aligned}
$$

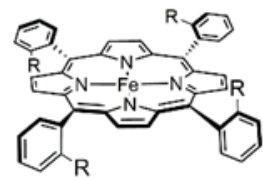

Complex

1

2

3

7

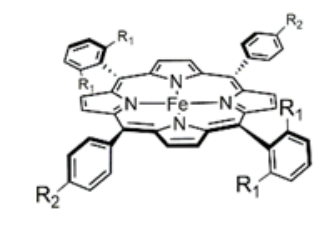

Complex $\quad R_{1} \quad R_{2}$

4

$\mathrm{H}_{3} \mathrm{C}$

$\mathrm{HN}=\mathrm{O} \quad-\mathrm{O}-\mathrm{CH}_{3}$

5

$\underset{\mathrm{HN}}{\mathrm{H}_{3} \mathrm{C}}=\mathrm{O} \quad-\mathrm{H}$

$\mathrm{H}_{3} \mathrm{C}$

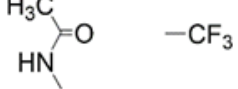

Scheme 1. The proposed mechanism of ORR catalyzed by Fe(TPP) and the structure of its derivatives studied here

according to the overall rate law (eq 7). This value is in good agreement with the rate constant, $3.4 \times 10^{6} \mathrm{M}^{-2} \mathrm{~S}^{-1}$ determined from spectroscopic measurements. ${ }^{16}$

The overall rate $=k_{\text {or }}\left[\mathrm{O}_{2}\right][\mathrm{FeTPP}]\left[\mathrm{H}^{+}\right]$

More detailed discussions for justifying these new interpretations and assignments can be found in SI. Here, how the inductive effect through the modifications of functional groups and the effect of the secondary coordination sphere on the faster chemical steps are further examined, particularly the relation between $k_{1}^{O R R}$ and the first reduction potential of

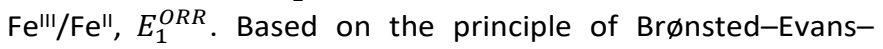
Polanyi, the correlation between $k_{1}^{O R R}$ and free energy between eq (3)-(5) ORR in Scheme 1 can be described as eq 8 as discussed before.22 Therefore, the effect of individual elementary chemical step proposed on $k_{1}^{O R R}$ can be evaluated quantitively if a correlation can be obtained as depicted in eq 8 .

$\frac{R T \ln 10}{F} \log k_{1}^{O R R}=-\alpha_{1} E_{1}^{O R R}-\alpha_{2} \frac{R T \ln 10}{F} p K_{a}+\alpha_{3} \frac{R T}{F} \ln K_{O_{2}}+C$

where $\alpha_{1}, \alpha_{2}$ and $\alpha_{3}$ are a correlation coefficient that is respectively associated with $E_{1}^{O R R}$ for redox couple of $\mathrm{Fe}$ "II/ $/ \mathrm{Fe}^{\prime \prime}$ , the $p K_{a}$ value for the acid used for the ORR and the binding constant, $K_{\mathrm{O}_{2}}$ between molecular oxygen and the Fe(TPP) derivative. $\mathrm{C}$ is a constant.

Fig 3 shows a linear correlation between $\log \left(k_{1}^{O R R}\right)$ and $E_{1}^{O R R}$ from complex 2-7 in the presence of $\mathrm{DMF}^{-\mathrm{H}^{+}}$as proton source, which suggests that these complexes operate within the same mechanism depicted for the faster chemical steps, eq (4)-(5) ORR. For complex 4-7, the complex with a more negative $E_{1}^{O R R}$ value, induced by electron-donating groups, facilitated the proton transfer step (eq (5) ORR), which follows the scaling relationship discussed earlier. Unexpectedly, the complex 2-3 modified with strong electron-withdrawing groups revealed a high overpotential with a high $k_{1}^{O R R}$ value. More interestingly, very recently, a reverse scaling relationship between $k_{1}^{O R R}$ and the free energy (i.e. the combination of $E_{1}^{O R R}$ and $p K_{a}$ ) has been found as shown in the red line in Fig 3 with the FeTPP derivative modified with a positive-charge trimethylanilinium group, $\mathbf{7}$ when various carboxylic acids were

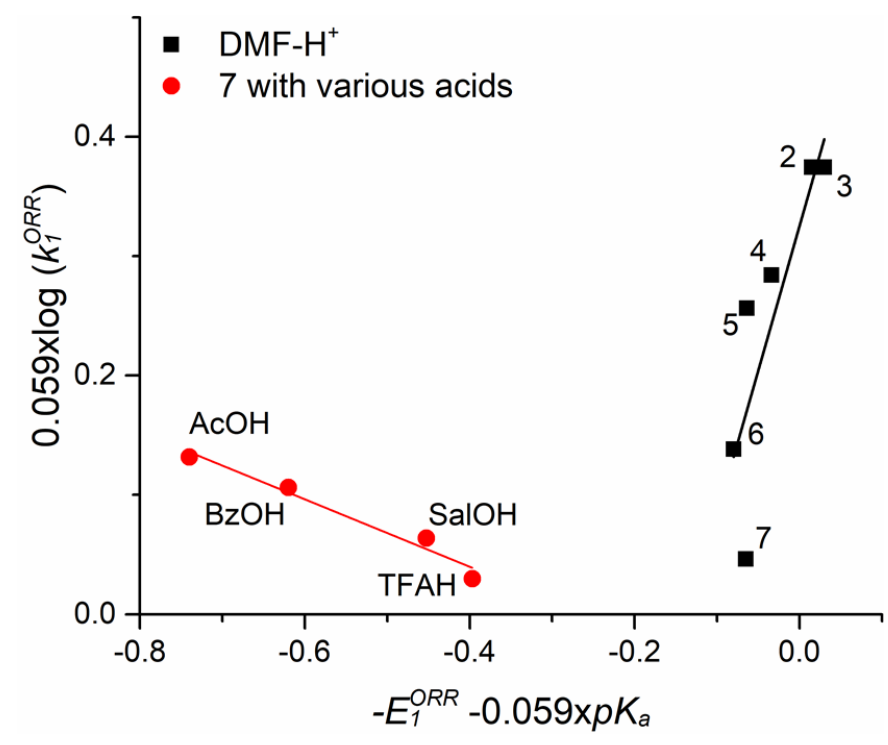

Fig 3. The relation between $\log \left(k_{1}^{O R R}\right)$ and the free energy for ORR catalyzed by FeTPP derivatives. The values for complex 2 6 are obtained from ref 17 and the values for complex 7 and red points are from ref 20 . For black points, $20 \mathrm{mM}$ [DMF-H]OTf $\left(p K_{a}=6.1\right.$ ) was used as proton source. For red data points, $0.1 \mathrm{M}$ buffer of $\mathrm{AcOH}$, acetic acid $\left(p K_{a}=23.5\right) ; \mathrm{BzOH}$, benzoic acid $\left(p K_{a}=21.5\right) ; \mathrm{SalOH}$, salicylic acid $\left(p K_{a}=16.7\right) ;$ and TFAH, trifluoroacetic acid $\left(p K_{a}=12.6\right)$ were used as proton source respectively and the complex 7 was employed as the catalyst for ORR. All data were collected in dimethylformamide (DMF) at 1 atm of $\mathrm{O}_{2}$

used as proton source. As expected, such modification yielded a more positive $E_{1}^{O R R}$ (the bottom-left point in the black line of Fig 3) with a small $k_{1}^{O R R}$ value, due to the withdrawing substituent, in the presence of non-carboxylic $\mathrm{DMF}^{-\mathrm{H}^{+}}$as proton resource. Nevertheless, the substituent greatly increased the binding affinity with the negative conjugate base ion that is associated with the carboxylic acids used for ORR. As 
a result, a much more negative $E_{1}^{O R R}$ value was required for the reactivation of conjugate-base bound $\mathbf{7}$ adduct for the reduction of a $\mathrm{Fe}(\mathrm{III}) / \mathrm{Fe}$ (II) redox couple and ORR, thereby increasing the $k_{1}^{O R R}$ value (red line in Fig 3). Interestingly, from the thermodynamic point of view, the effect on $k_{1}^{O R R}$ from the more negative reduction potential required for the conjugate base-7 adduct complex outweighs a lower driving force from a much weaker carboxylic acid used compared to DMF- $\mathrm{H}^{+}$(The $\mathrm{pK}_{\mathrm{a}}$ value for acid shown in Fig 3 caption). Finally, it is worth pointing out that the rate law for the overall reaction is equal to the combination of eq (3)-(5) ORR in Scheme $1 .{ }^{16}$ Nevertheless, it is interesting to examine how these derivatives affect the ratelimiting step, $\mathrm{O}-\mathrm{O}$ bond breaking in the future, particularly with the modification of the carboxylate groups which aim to act as proton relay or stabilize the intermediate species through hydrogen-bonding interactions.

\section{Case B. $\mathrm{CO}_{2}$ reduction reaction}

To further demonstrate how the key feature of voltammograms and FOWA plots can be useful to gain mechanistic insights, we briefly compare case A to the same Fe(TPP) complex that has also been displayed to catalyze the reduction of $\mathrm{CO}_{2}$ to $\mathrm{CO}$ by Savéant, Costentin, Robert and their co-workers. 15, 21, 23-28 The catalytic reaction take places after the reduction of $\mathrm{Fe}(\mathrm{I})$ to $\mathrm{Fe}(0)$ in $\mathrm{Fe}(T P P)$ as shown in Scheme 2 and the reaction mechanism follows $E C{ }^{\text {Homo }} \mathrm{C}$. As demonstrated in a series of papers from these authors, ${ }^{21}, 24$ the FOWA plots obtained from these derivatives exhibited a straight line, which indicates that the apparent rate constant, $k_{1}^{C R R}$ determined by FOWA is the ratelimiting step as a function of rate constants collectively combined from eq (4)-(6) $)_{\mathrm{CRR}}$ in Scheme 2. According to Table 1 , the apparent rate constant for the faster chemical step, $k_{2}^{C R R}$ can be ascribed to the rapid release of CO product (eq (7) CRR $_{\text {). }}$.

In addition, a correlation between $\log \left(k_{1}^{C R R}\right)$ and $E_{1}^{C R R}$ among FeTPP derivatives was found as shown in Fig $4{ }^{21,28}$ where the black line exhibits a series of fluorine-substituted FeTPP derivatives (8-10, the corresponding chemical structures can be found in $\mathrm{SI}$ ). As a number of fluorine atoms incorporated into the phenyl group increased, the $E_{1}^{C R R}$ value shifted toward more positive due to a stronger electron-withdrawing property but the corresponding rate constant became smaller. ${ }^{28}$ Two different approaches have been applied to exploit the effect of SCS on complex 1: hydrogen-bonding/proton relay (red line) ${ }^{24-}$ ${ }^{26}$ and electrostatic interactions (blue line). ${ }^{27}$ For the hydrogenbonding interactions/proton relay, the introduction of hydroxyphenyl substituents to $\mathrm{Fe}(\mathrm{TPP}), \mathbf{1 1 - 1 2}$, have been

$$
\begin{array}{rlll}
\mathrm{CO}_{2}+2 \mathrm{e}^{-}+2 \mathrm{HA} & \stackrel{\text { TPP-Fe }}{\longrightarrow} \mathrm{CO}+2 \mathrm{H}_{2} \mathrm{O}+2 \mathrm{~A}^{-} & & (1)_{\mathrm{CRR}} \\
\mathrm{HA} & \rightleftharpoons \mathrm{H}^{+}+\mathrm{A}^{-} & \mathrm{Ka}_{\mathrm{a}} & { }^{(2)_{\mathrm{CRR}}}
\end{array}
$$

Mechanism

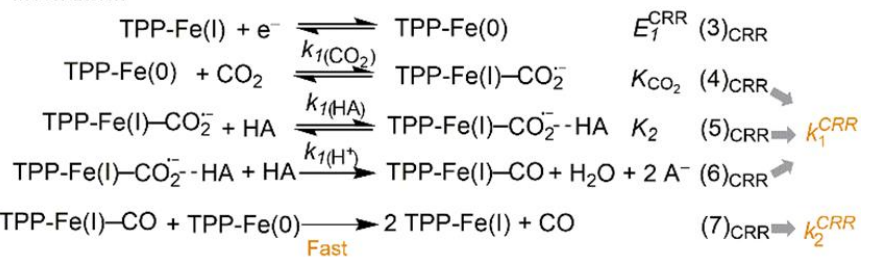

Scheme 2. The mechanism of CRR catalyzed by Fe(TPP).

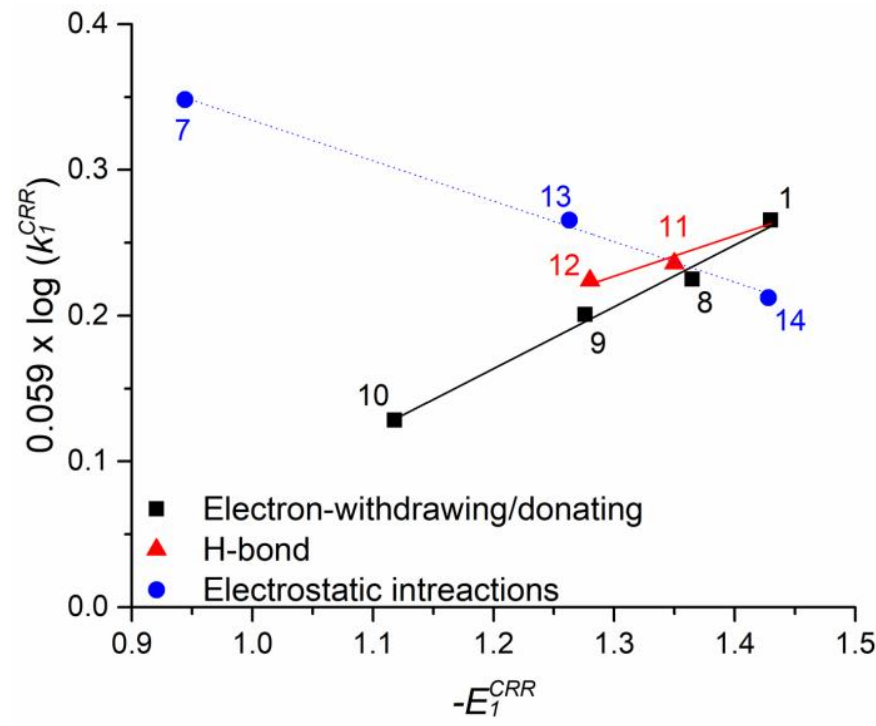

Fig 4. The relation between $\log \left(k_{1}^{C R R}\right)$ and $E_{1}^{C R R}$ among $\mathrm{Fe}(\mathrm{TPP})$ derivatives. The values of $\mathbf{1}$ is obtained from ref 21 . The values of 8 $\mathbf{1 0}$ is obtained from ref 28 . The values of $\mathbf{1 1 - 1 2}$ is obtained from ref 25-26. The value of 7 and $\mathbf{1 3 - 1 4}$ is obtained from ref 27 . All experimental conditions were performed with $0.23 \mathrm{M} \mathrm{CO}_{2}$ and $3 \mathrm{M}$ phenol in DMF. The $E_{1}^{C R R}$ value for $\mathrm{Fe}(\mathrm{l}) / \mathrm{Fe}(0)$ redox couple is determined in the absence of $\mathrm{CO}_{2}$ and phenol. The corresponding chemical structure can be found in SI.

shown to stabilize the $\mathrm{CO}_{2}$-bound intermediate species through hydrogen-bonding. Consequently, the catalytic rate increased (blue line vs black line) although the mechanism is different from scheme $2 .{ }^{25}$ In comparison, the modification of charge substituents on the phenyl group of FeTPP $(\mathbf{7}, \mathbf{1 3 - 1 4})$ to stabilize the negative-charge intermediate state (eq (4)-(5) CRR) through the electrostatic interactions revealed a remarkable enhancement of catalytic activity. ${ }^{27}$ The positive-charge substituent derivatives ( 7 and 13 ) not only shifted $E_{1}^{C R R}$ to be more positive but also increased the rate constant as opposed to the negative-charge substituent, 14. More interestingly, the strategy through electrostatic interactions seemingly reveal a reverse scaling relationship. The complex $\mathbf{7}$ stands as the best Fe porphyrin complex for $\mathrm{CO}_{2}$ reduction to $\mathrm{CO}$ reported in literature in terms of the rate with a given overpotential reported so far. ${ }^{29}$

\section{Case C. Hydrogen evolution Reaction (HER)}

Inspired by [FeFe] hydrogenases, the $\left[\mathrm{Ni}-\left(\mathrm{PR}_{2} \mathrm{NR}_{2}{ }_{2}\right)_{2}\right]^{2+}$ derivatives developed by the Dubois group and then Pacific Northwest National Laboratory demonstrated that the introduction of pendant amines as proton relay into the $\mathrm{Ni}$-bis(diphosphine) derivatives plays a key role in enhancing the activity of HER and hydrogen oxidation reaction. ${ }^{5,6}, 10,30-34$ As blue highlighted in Scheme 3 , the catalytic cycle for $\mathrm{H}_{2}$ evolution has been proposed through ECEC mechanism with $k_{1}^{H E R}>k_{2}^{H E R}$. Here, the apparent rate constant, $k_{1}^{H E R}$ and $k_{2}^{H E R}$ can be regarded as a function of a collection of the rate constants of proposed productive and unproductive steps as exhibited in Scheme 3. More recently, the EECC mechanism has been further suggested to occur when a high overpotential is applied. ${ }^{10,35,36}$ The S- 


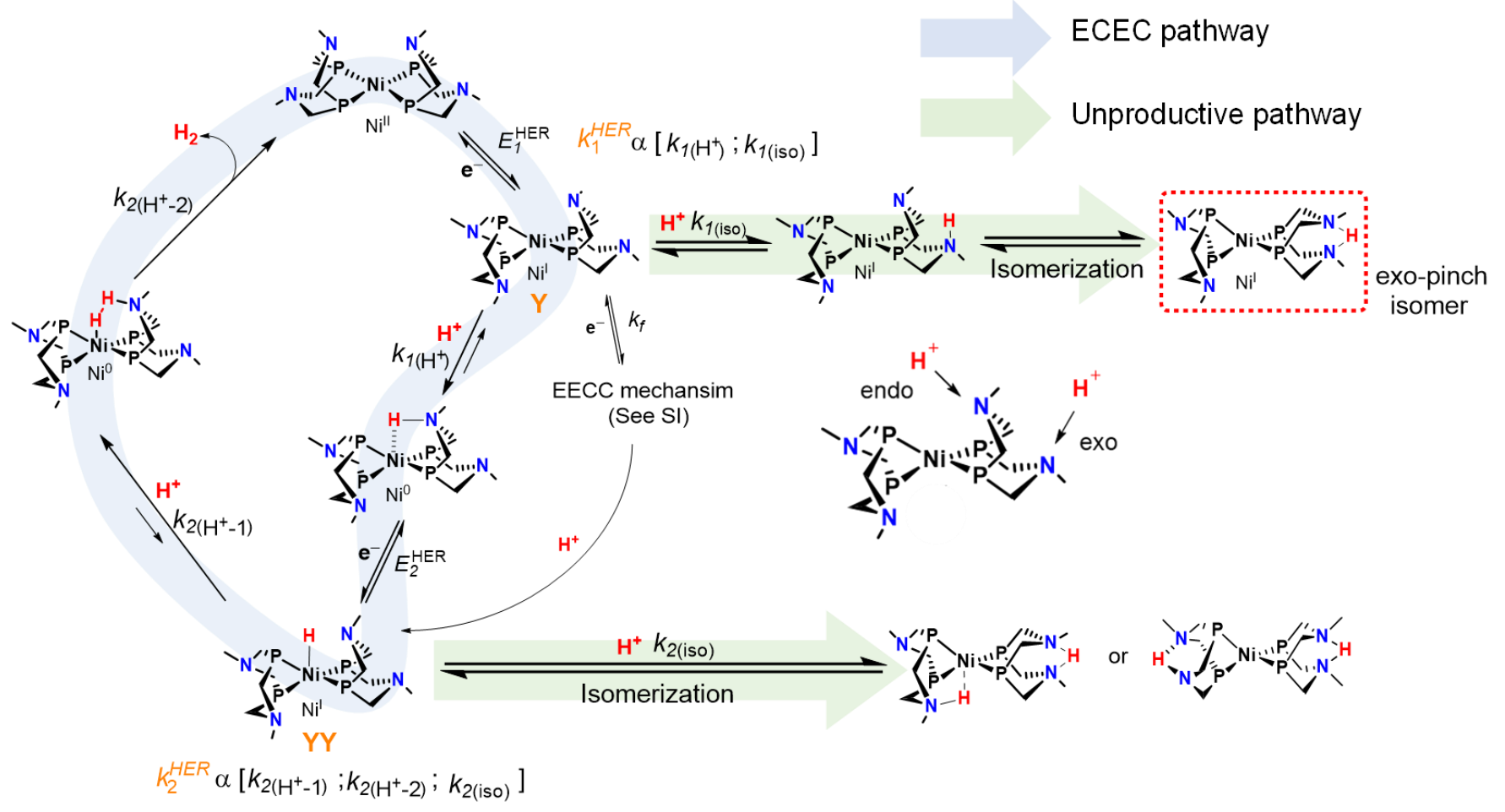

Scheme 3. The ECEC mechanism of HER mediated by [Ni-( $\left.\mathbf{P R}_{2} \mathbf{N}^{\mathbf{R}^{\prime}}\right)_{2}$ ]. The blue highlight refers to the ECEC mechanism for HER. The green highlights refer to the unproductive pathways that compete with the ECEC mechanism to generate the inactive pinched isomers (e.g. the species squared by the red dot) via protonation on the exo-position $\mathrm{N}$ atom. For clarity, the detailed mechanism of EECC is omitted and the full proposed mechanism can be found in the SI. The contribution of $k_{f}$ from the EECC mechanism on $k_{1}^{H E R}$ is negligible because ECEC is the dominant mechanism discussed here.

shape electrocatalytic voltammograms were obtained for most of the $\left[\mathrm{Ni}-\left(\mathrm{PR}_{2} \mathrm{~N}^{\mathrm{R}^{\prime}}\right)_{2}\right]^{2+}$ derivatives studied under the substratesaturated condition, which allows directly to estimate the apparent rate constant for the rate-limiting step, $k_{2}^{H E R}$ through $i_{\text {lim. }}$. For the faster apparent rate constant, $k_{1}^{H E R}$, the value can be estimated through eq T3 in Table 1 with the input of reported $E_{\text {cat } / 2}^{H E R}$ and $k_{2}^{H E R}$ values. Although the EECC mechanism is the dominant pathway for HER near the region of $i_{\text {lim }}$, the apparent rate constant, $k_{2}^{H E R}$, represented for a collection of the pathways for the second chemical step between the ECEC and EECC mechanism, is the same (i.e. the same reaction pathway after the key intermediate $Y Y$ as shown in scheme 3 ). Therefore, the $k_{2}^{H E R}$ value is likely to be similar between two mechanisms, which has been shown recently. ${ }^{10}$ In addition, the half-wave potential $E_{c a t / 2}^{H E R}$ has been shown to be only associated with the ECEC mechanism. ${ }^{10}$

As shown in Scheme 3, the branch and unproductive pathways (green highlights) participating with the protonation process at the exo position of pendant amine than the endo site, have been known to be detrimental to catalytic activity. ${ }^{37}$ The boat-chair isomerization occurs through intramolecular proton transfer to generate the inactive exo-pitch protonated species (as shown by the red-dot square in scheme 3), which is kinetically favored and thermodynamically more stable compared to the active endo protonated species. 6,3537 Therefore, the $k_{1}^{H E R}$ and $k_{2}^{H E R}$ value, as a function of these isomerization pathways (i.e. $k_{1 \text { (iso) }}$ and $k_{2 \text { (iso) }}$ shown in Scheme 3 respectively), are expected to reflect the influence of these undesired pathways.

To further explore the influence of the active site and SCS on the electrocatalytic mechanism for HER mediated by the [Ni$\left(\mathrm{P}_{2} \mathrm{~N}^{\mathrm{R}_{2}}{ }_{2}\right)_{2}$ ] complex, the modifications of the parent complex 15 were conducted in two different directions: the substituent on the $\mathrm{P}$ atom that coordinates to the $\mathrm{Ni}$ atom, is supposed to directly manipulate the electronic and chemical properties of the active site. ${ }^{31,} 32$ In comparison, the modifications on the $\mathrm{N}$ atom of the $\left[\mathrm{Ni}-\left(\mathrm{P}_{2}{ }_{2} \mathrm{~N}^{\prime}{ }_{2}\right)_{2}\right]$ complex could lead to further clues on the role of the secondary coordination sphere. ${ }^{30}$ As the black line shown in Fig $5 b$, the modifications of phosphine with less bulky substituents, 16 and 17 (Fig 6) yielded to the more negative $E_{1}^{H E R}$, which is associated with the higher $k_{2}^{H E R}$ value compared to bulky substitutions, 18 and 19. As opposed, when varying the acidity of pendant amines through electrondonating/withdrawing substitutions at the para position of the phenyl group (20-23), the $k_{2}^{H E R}$ value increased as the $p K_{a}$ value of the protonated pendant amine decreased. ${ }^{30}$ Intriguingly, at the same time, due to the inductive effect of these substitutions, the more electron-withdrawing derivative led to shift $E_{1}^{H E R}$ (e.g. 23) toward the more positive value. Therefore, a reverse linear relationship is obtained as shown in the red line in Fig 5b. In contrast, no apparent (or weak) correlation between $E_{1}^{H E R}$ and $k_{1}^{H E R}$, is found as shown in Fig 5a. Overall, the enhancement of the overall activity through these modifications were not apparent as compared to the parent complex 15 and the difference between $k_{1}^{H E R}$ and $k_{2}^{H E R}$ in the 

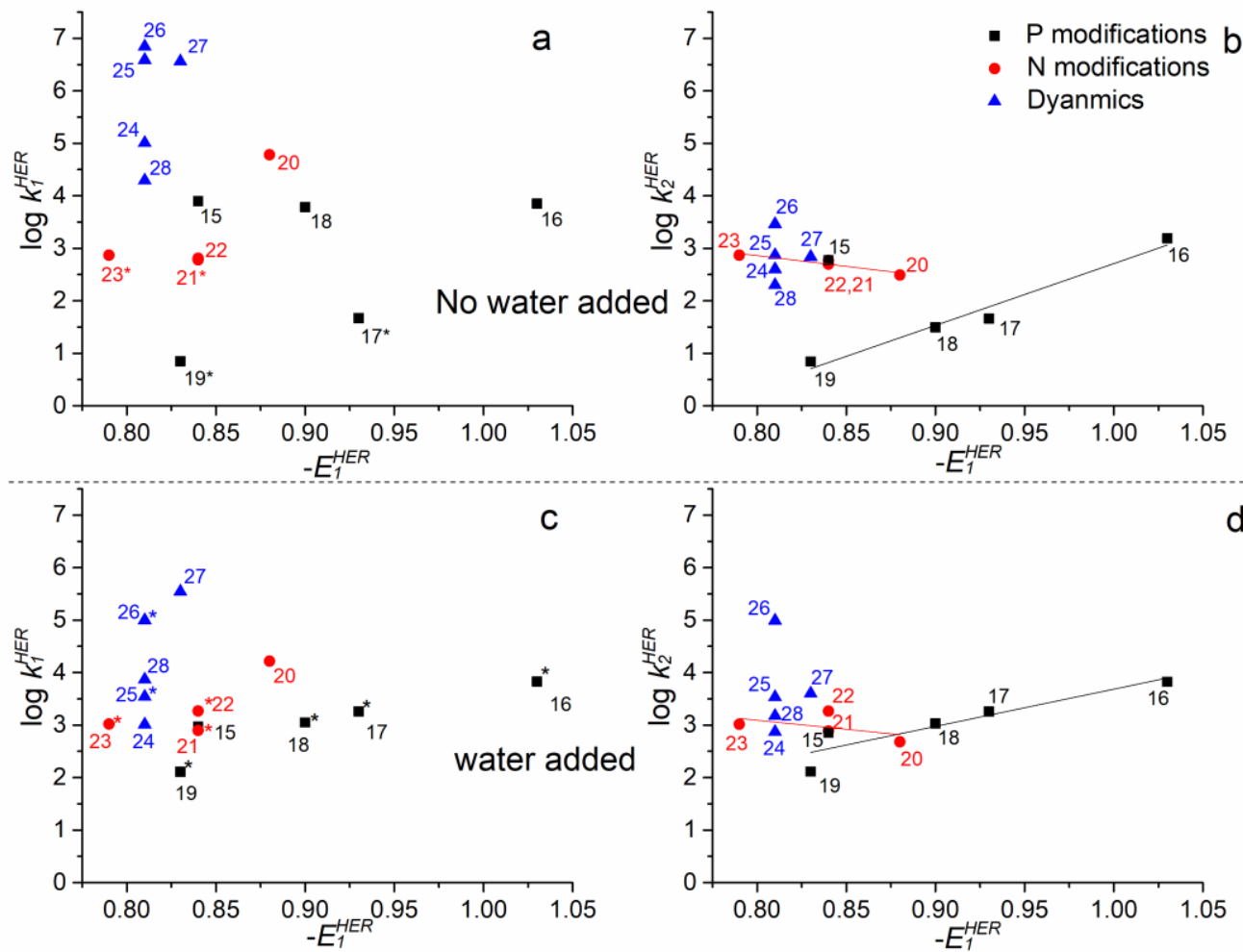

Fig 5. The relation between $\log \left(k_{1}^{H E R}\right) / \log \left(k_{2}^{H E R}\right)$ and $E_{1}^{H E R}$ among $\left[\mathrm{Ni}-\left(\mathrm{PR}_{2} \mathrm{~N}^{\mathrm{R}^{\prime}}{ }_{2}\right)_{2}\right]^{2+}$ derivatives in the absence of water $(\mathrm{a}, \mathrm{b})$ and presence of water $(\mathbf{c}, \mathbf{d})$. The $k_{2}^{H E R}$ and $E_{\text {cat } / 2}^{H E R}$ values of the Ni complexes were collected from literature as below: complex 15 from ref 5; complex 16 from ref 32; complex 17-19 from ref 31; complex 20-23 from ref 30 and complex 24-28 from ref 33. All the values were collected in dry acetonitrile using DMF- $\mathrm{H}^{+}$as proton source. The asterisk sign indicates that the reported value, $E_{\text {cat } / 2}^{H E R}$ is equal or slightly negative than $E_{1}^{H E R}$, which suggests the difference between the two apparent rate constants is very small. Therefore, the value for $k_{1}^{H E R}$ is assumed to be the same as $k_{2}^{H E R}$.

most derivatives is around the range of an order of magnitude. These results are probably due to the influence of the unproductive processes for the formation of the inactive exopitch protonated species. According to theoretical calculations, these inactive pathways are kinetically more favorable for both key intermediate species $Y$ and $Y Y$ in Scheme 3. 3335

To decrease the rate of the unproductive pathways, recently, the long $\mathrm{n}$-alky chains and bulky substitutions on [Ni$\left.\left(\mathrm{P}_{2} \mathrm{~N}^{\mathrm{R}_{2}}\right)_{2}\right]$ (24-28) were incorporated at the para position of the phenyl group of the $\mathrm{N}$ atom of the parent complex 15, to minimize the process of chair-boat isomerization processes through the steric effects without greatly affecting the electronic structure of the active site (i.e. the reduction potential of $E_{1}^{H E R}$ of 24-28 compared to 15). ${ }^{33}$ Consequently, the rate constant $k_{2}^{H E R}$ for these substituents increased were observed with a lower overpotential required for HER (blue dots in Fig 5b). In comparison, such modifications have much more prominent impact on $k_{1}^{H E R}$, where the values increased with several orders of magnitude compared to the modifications described in the previous section (i.e. blue dots vs black/red dots in Fig 5a). These results suggest that the intermediate species, $\mathrm{Y}$ in Scheme 3 is greater affected by the unproductive protonation processes at the exo position than the intermediate species, YY.

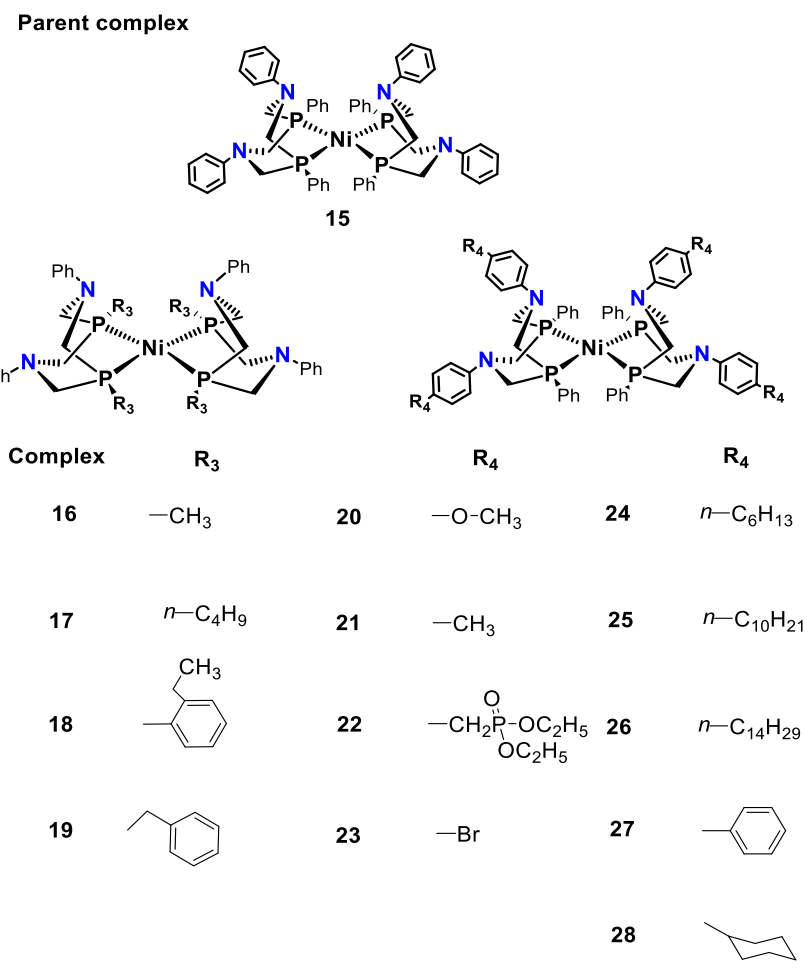

Fig 6. The chemical structure of $\left[\mathrm{Ni}-\left(\mathrm{P}^{\mathrm{R}}{ }_{2} \mathrm{~N}^{\mathrm{R}^{\prime}}{ }_{2}\right)_{2}\right]$ 
Finally, the addition of protic solvent as cosolvent to facilitate proton transfer was found to further enhance catalytic activity. For example, in the presence of water as cosolvent, the $k_{2}^{H E R}$ for the rate-limiting step increased as shown in Fig $5 \mathrm{~d}$ compared to Fig $5 \mathrm{~b}$. However, the more negative $E_{c a t / 2}^{H E R}$ values (i.e. higher overpotential) were observed concomitantly in the presence of water, which indicates that the difference between $k_{1}^{H E R}$ and $k_{2}^{H E R}$ becomes small. For the most derivatives, the $k_{1}^{H E R}$ values are very close to $k_{2}^{H E R}$, suggesting that unproductive protonation processes associated with $k_{1 \text { (iso) }}$ are accelerated in the presence of cosolvent as well, which show no apparent effect on $k_{1}^{H E R}$ values compared to the absence of cosolvent. In brief, these two strategies highlight the importance of the roles even beyond the secondary coordination sphere, which could have different impacts on different chemical steps.

\section{Discussion}

Here, we show that the rate constants for different chemical steps of multi-electron redox reactions can be disentangled by the analysis of electrocatalytic voltammograms and FOWA plots, in which the correlation between the apparent rate constants and thermodynamic parameters can be examined. Particularly, unlike a general conception in the community, FOWA actually leads to the rate constant for a faster chemical step rather than a rate-limiting step in some kinetic scenarios (Table 1). Therefore, the role of the SCS and beyond on the faster chemical steps for fuel-forming reactions can be further examined, which provide quantitative structure activity relationship. Although the value evaluated here are apparent rate constants, the elaborate experimental designs (such as variation of substrate/cosubstrate concentration) with kinetic analysis allow to further determine the intrinsic rate constant related to the corresponding elementary step proposed. Such practices have been recently showcased for FeTPP derivatives for CRR reaction $21,25,26$ and MnTPP for ORR ${ }^{38}$. In addition, although the redox reactions investigated here are involved with the ECEC-type mechanism, other kinetic mechanisms (such as EECC) have been shown to have their own characteristic features of FOWA plots that are also associated with the specific kinetic phenomena. ${ }^{8,9}$

As for the fuel-forming reactions investigated here, the results suggest that not all chemical steps are facilitated through the modification of the SCS and directly correlated with the corresponding reduction potential. For example, it would be expected that the $k_{1}^{H E R}$ value of a series of $\left[\mathrm{Ni}-\left(\mathrm{P}_{2} \mathrm{~N}^{\mathrm{R}_{2}}{ }_{2}\right)_{2}\right]^{2+}$ derivatives should have a correlation with the corresponding $E_{1}^{H E R}$ as observing a linear relation between $k_{1}^{O R R}$ and $E_{1}^{O R R}$ in ORR. The lack of a correlation between $k_{1}^{H E R}$ and $E_{1}^{H E R}$ can be ascribed to the inactive protonation processes for $[\mathrm{Ni}$ $\left.\left(\mathrm{P}_{2} \mathrm{~N}^{\mathrm{R}_{2}}{ }_{2}\right)_{2}\right]^{2+}$ derivatives, which greatly disrupts the expected relation between kinetic and thermodynamic values. On the other hand, finding a free energy relationship between $k_{1}^{O R R}$ and $E_{1}^{O R R}$ can provide useful guidelines to further modify the SCS to facilitate chemical steps beyond the rate-limiting step. Overall, these kinetic analyses can help dissect chemical steps to broaden the understanding of catalytic mechanism into catalyst optimization.

Finally, how do lessons learned from homogeneous molecular electrocatalysts translate to heterogeneous thin-film electrocatalysts? For most of thin-film electrocatalysts, the scaling relationships found are derived from the studies of the active site alone without considering the effect of the SCS and beyond. ${ }^{39-43}$ In terms of the catalytic mechanism, both systems are expected to share similar essential pathways on the atomic level. Finding key features from the studies of molecular systems with the influence of the SCS and beyond can provide new insights to further tailor thin-film electrocatalysts to break down the scaling relationship. For example, recently, the presence of a high concentration of cations in the reaction environment has shown to greatly facilitate the electrocatalytic activity of heterogeneous metal electrocatalysts for $\mathrm{CO}_{2}$ reduction due to electric field interaction provided by these ions to stabilize the intermediate species. ${ }^{44-47}$ Such influence can be regarded as the effect of the secondary coordination sphere, which is similar to the results showcased from case B discussed here. Nevertheless, the key success to transform these findings to electrocatalysts lies in introducing a right kind of interaction from the SCS and in locating it at the relevant distance to effectively optimize the active site for electron transfer and chemical steps. At the moment, knowledge and predictive power on these aspects for these fuel-forming reactions is still lacking. Therefore, molecular electrocatalysts play an important frontline role in exploring these uncharted territories.

\section{Conclusion}

In brief, we demonstrate how kinetic analysis through S-shape electrocatalytic voltammograms and FOWA plots can be applied to identify reaction mechanism and determine rate constants for multi-electron redox reaction. Therefore, the influence of the SCS and beyond on the separately chemical steps are able to further explore quantitatively, revealing how these additional interactions affect the different stages of the reaction. The results and analysis demonstrated here provide further evaluations of the effect of these supernumerary interactions and fresh ideas for the optimization of a desired electrocatalyst to facilitate fuel-forming reaction beyond the active site.

\section{Conflicts of interest}

There are no conflicts to declare.

\section{Acknowledgement}

We are very grateful to Prof. Sunney Chan (Caltech/Academia Sincia) and Msc. Robin Tyburski (Uppsala University) for critical comments and suggestions on the manuscript. Dr. Ding-Chi Huang (China Petrochemical Development Cooperation), is thankful for assisting preparations of some graphics of chemical structures and schemes. 


\section{References}

1.

Z. W. Seh, J. Kibsgaard, C. F. Dickens, I. Chorkendorff, J. K. Nørskov and T. F. Jaramillo, Science, 2017, 355, eaad4998.

2. K. E. Dalle, J. Warnan, J. J. Leung, B. Reuillard, I. S. Karmel and E. Reisner, Chem. Rev., 2019, 119, 2752-2875.

3. C. Costentin and J.-M. Savéant, Nat. Rev. Chem, 2017, 1, 0087.

4. F. A. Armstrong and J. Hirst, Proc. Natl. Acad. Sci. U.S.A, 2011, 108, 14049-14054.

5. A. D. Wilson, R. H. Newell, M. J. McNevin, J. T. Muckerman M. Rakowski DuBois and D. L. DuBois, J. Am. Chem. Soc., 2006, 128, 358-366.

6. W. J. Shaw, M. L. Helm and D. L. DuBois, Biochimica et Biophysica Acta (BBA) - Bioenergetics, 2013, 1827, 11231139.

7. S. Chatterjee, K. Sengupta, B. Mondal, S. Dey and A. Dey, Acc. Chem. Res., 2017, 50, 1744-1753.

8. C. Costentin and J.-M. Savéant, ChemElectroChem, 2014, 1, 1226-1236.

9. V. C. C. Wang and B. A. Johnson, ACS Catal., 2019, 9, 71097123.

10. E. S. Wiedner, H. J. S. Brown and M. L. Helm, J. Am. Chem. Soc., 2016, 138, 604-616.

11. E. S. Rountree, B. D. McCarthy, T. T. Eisenhart and J. L. Dempsey, Inorg. Chem., 2014, 53, 9983-10002.

12 A. M. Appel and M. L. Helm, ACS Catal., 2014, 4, 630-633. R. S. Nicholson and I. Shain, Anal. Chem., 1964, 36, 706723.

J. M. Saveant and E. Vianello, Electrochim. Acta, 1965, 10, 905-920.

15. C. Costentin, S. Drouet, M. Robert and J.-M. Savéant, J. Am. Chem. Soc., 2012, 134, 11235-11242.

$16 . \quad$ D. J. Wasylenko, C. Rodríguez, M. L. Pegis and J. M. Mayer J. Am. Chem. Soc., 2014, 136, 12544-12547.

M. L. Pegis, B. A. McKeown, N. Kumar, K. Lang, D. J. Wasylenko, X. P. Zhang, S. Raugei and J. M. Mayer, ACS Central Science, 2016, 2, 850-856.

18 M. L. Pegis, C. F. Wise, B. Koronkiewicz and J. M. Mayer, J. Am. Chem. Soc., 2017, 139, 11000-11003.

19. M. L. Pegis, D. J. Martin, C. F. Wise, A. C. Brezny, S. I. Johnson, L. E. Johnson, N. Kumar, S. Raugei and J. M. Mayer, J. Am. Chem. Soc., 2019, 141, 8315-8326.

20. D. J. Martin, B. Q. Mercado and J. M. Mayer, Science Advances, 2020, 6, eaaz3318.

21. C. Costentin, S. Drouet, G. Passard, M. Robert and J.-M. Savéant, J. Am. Chem. Soc., 2013, 135, 9023-9031.

22 C. Costentin and J.-M. Savéant, J. Am. Chem. Soc., 2018, 140, 16669-16675.

23. I. Bhugun, D. Lexa and J.-M. Savéant, J. Am. Chem. Soc., 1996, 118, 1769-1776.

24. C. Costentin, S. Drouet, M. Robert and J.-M. Savéant Science, 2012, 338, 90-94.

25.

C. Costentin, G. Passard, M. Robert and J.-M. Savéant, J. Am. Chem. Soc., 2014, 136, 11821-11829.

26. C. Costentin, G. Passard, M. Robert and J.-M. Savéant, Proc. Natl. Acad. Sci. U.S.A, 2014, 111, 14990-14994.

27. I. Azcarate, C. Costentin, M. Robert and J.-M. Savéant, J. Am. Chem. Soc., 2016, 138, 16639-16644.

28. I. Azcarate, C. Costentin, M. Robert and J.-M. Savéant, J. Phys. Chem. C, 2016, 120, 28951-28960.
29. P. Gotico, Z. Halime and A. Aukauloo, dalton trans., 2020, 49, 2381-2396.

30. U. J. Kilgore, J. A. S. Roberts, D. H. Pool, A. M. Appel, M. P. Stewart, M. R. DuBois, W. G. Dougherty, W. S. Kassel, R. M. Bullock and D. L. DuBois, J. Am. Chem. Soc., 2011, 133, 5861-5872.

31. U. J. Kilgore, M. P. Stewart, M. L. Helm, W. G. Dougherty, W. S. Kassel, M. R. DuBois, D. L. DuBois and R. M. Bullock, Inorg. Chem., 2011, 50, 10908-10918.

32. S. Wiese, U. J. Kilgore, D. L. DuBois and R. M. Bullock, ACS Catal., 2012, 2, 720-727.

33. A. J. P. Cardenas, B. Ginovska, N. Kumar, J. Hou, S. Raugei, M. L. Helm, A. M. Appel, R. M. Bullock and M. O'Hagan, Angew. Chem. Int. Ed., 2016, 55, 13509-13513.

34. C. M. Klug, A. J. P. Cardenas, R. M. Bullock, M. O'Hagan and E. S. Wiedner, ACS Catal., 2018, 8, 3286-3296.

35. M.-H. Ho, R. Rousseau, J. A. S. Roberts, E. S. Wiedner, M. Dupuis, D. L. DuBois, R. M. Bullock and S. Raugei, ACS Catal., 2015, 5, 5436-5452.

E. S. Rountree and J. L. Dempsey, J. Am. Chem. Soc., 2015, 137, 13371-13380.

M. O'Hagan, M.-H. Ho, J. Y. Yang, A. M. Appel, M. R. DuBois, S. Raugei, W. J. Shaw, D. L. DuBois and R. M. Bullock, J. Am. Chem. Soc., 2012, 134, 19409-19424.

38. G. Passard, D. K. Dogutan, M. Qiu, C. Costentin and D. G. Nocera, ACS Catal., 2018, 8, 8671-8679.

39. J. K. Nørskov, J. Rossmeisl, A. Logadottir, L. Lindqvist, J. R. Kitchin, T. Bligaard and H. Jónsson, J. Phys. Chem. B, 2004, 108, 17886-17892.

40. I. C. Man, H.-Y. Su, F. Calle-Vallejo, H. A. Hansen, J. I. Martínez, N. G. Inoglu, J. Kitchin, T. F. Jaramillo, J. K. Nørskov and J. Rossmeisl, ChemCatChem, 2011, 3, 11591165.

41. H. A. Hansen, J. B. Varley, A. A. Peterson and J. K. Nørskov, The Journal of Physical Chemistry Letters, 2013, 4, 388-392. V. C. C. Wang, PCCP, 2016, 18, 22364-22372.

43. K. S. Exner and H. Over, Acc. Chem. Res., 2017, 50, 12401247.

44. M. R. Singh, Y. Kwon, Y. Lum, J. W. Ager and A. T. Bell, J. Am. Chem. Soc., 2016, 138, 13006-13012.

45. M. Liu, Y. Pang, B. Zhang, P. De Luna, O. Voznyy, J. Xu, X. Zheng, C. T. Dinh, F. Fan, C. Cao, F. P. G. de Arquer, T. S. Safaei, A. Mepham, A. Klinkova, E. Kumacheva, T. Filleter, D. Sinton, S. O. Kelley and E. H. Sargent, Nature, 2016, 537, 382-386.

46. E. Pérez-Gallent, G. Marcandalli, M. C. Figueiredo, F. CalleVallejo and M. T. M. Koper, J. Am. Chem. Soc., 2017, 139, 16412-16419.

47. S. Ringe, E. L. Clark, J. Resasco, A. Walton, B. Seger, A. T. Bell and K. Chan, Energy \& Environmental Science, 2019, 12, 3001-3014. 
TOC Figure

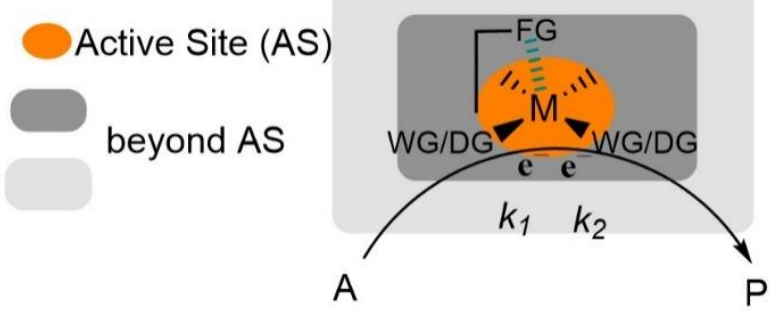

